están en las casas de mi morada respecto de que los susodichos an vivido y estan en mi cargo y asi mismo los e sustentado y alimentado $t^{0}$ de tres años (...) declarolo así para conste dello.

Yten conformandome con las leyes destos reinos desde luego mejor e por mejorada a la dha $\mathrm{d}^{\mathrm{a}} \mathrm{Fran}^{\mathrm{ca}}$ Velazquez mi hija mujer lixitima del dho Carlos de Santamaría en este y remanente doy quinto de mis vienes muebles e raices deudas y acciones por el amor que la tengo y por aberme sido obediente y acudidome a todas mis necesidades y enfermedades y por que a desde mi $\mathrm{v}^{\mathrm{d}}$ (demás disposiciones)

Yten declaro que para executar y cumplir con mi testamento nombro por mis albaceas a Carlos de Santa María y a don Diego Carrillo clerigo presb ${ }^{\circ}$ desta ciudad

Yten mando a doña ynes luisa mi sobrina de edad de diez años quatro sillas negras de vaqueta y una media cama de madera colorada y dorada y cien reales de plata los cuales se entreguen a da María de Saravuia su madre.

Yten mando que todos mis bienes muebles sean sacados a subasta pública. Nombro por herederos universales a Diego Velazquez ayuda de camara de su majestad y a $\mathrm{d}^{\mathrm{a}}$ Fran $^{\mathrm{ca}}$ Velazquez hijos legitimos y a Cristobal de la Cueva mi nieto hijo legitimo de Juan Velazquez difunto y Maria de la Cueva (...)».

Archivo Histórico Provincial. Sección Protocolos Notariales, legajo 532, ff. 393-394v.

\title{
LOS TASADORES DIEGO VELÁZQUEZ Y JUANA PACHECO
}

El año 1999 conmemora el IV centenario del nacimiento de Diego de Silva y V̧êlázquez, uno de nuestros más geniales e insignes pintores de todos los tiempos de la historia universal. La búsqueda documental de nuevas fuentes que puedan enriquecer la historia de la pintura española, unida a una pequeña dosis de casualidad, ha querido que realicemos una novedosa aportación sobre algún aspecto biográfico y laboral de un personaje al que, por la amplitud de la bibliografía sobre él ya existente, parece que poco restaba por añadir ${ }^{1}$.

Este nuevo hallazgo nos permite descubir dos vertientes poco comunes en la hasta ahora conocida trayectoria del artista: una, como tasador de pinturas ${ }^{2}$; otra, y por primera vez para nosotros, trabajando bajo un mismo encargo y de manera conjunta al lado de su mujer Juana Pacheco.

Lo que aquí vamos a tratar ocurrió en Madrid durante 1636. Es el período entre su primer y segundo viaje a Italia. Un tiempo en el que la llamada Torre de la Parada, camino de El Pardo madrileño, se encuentra a punto de engalanarse con los lienzos pintados por Velázquez para tal regio encargo. Probablemente el pintor debía estar finalizando con destino a este mismo lugar el retrato venatorio del príncipe Baltasar Carlos, pintado al parecer entre los meses de octubre de 1635-1636 ${ }^{3}$. Recordemos que ese año Velázquez, pintor del rey hacía ya más de una década, fue nombrado Ayuda de Guardarropa de Su Majestad ${ }^{4}$. Tres me-

1 El documento base de este artículo, fue citado por Peter Cherry: New documents for Velazquez in the 1620s. En «The Burlington Magazine», vol. CXXXIII, n. ${ }^{\circ} 1.055$, febrero 1991, pp. 108-115, nota n. ${ }^{\circ} 44$.

2 Veáse J. López Navío: Velázquez tasa los cuadros de su protector D. Juan de Fonseca. En «Archivo Español de Arte», 1961, tomo XXXIV, pp. 53-84.

3 Véase cat. exp. Velázquez. Museo del Prado, del 23 de enero al 31 de marzo; n. ${ }^{\circ} 45$, pp. 276-281. Madrid, 1990.

${ }^{4}$ Con fecha 28 de julio de 1636. Véase Rodríguez Villa, Antonio: La Corte y Monarquía de España en sus años de 1636 y 1637 con curiosos documentos sobre corridas de toros de los siglos XVII y XVIII. Madrid, 1886, pp. $27-28$. 
ses más tarde, presentó al rey Felipe IV un memorial solicitando el pago de algunos atrasos a deber ${ }^{5}$. El año se completa a nivel documental con una información durante el mes de abril ${ }^{6}$, en la que Velázquez realiza la tasación que más adelante nos viene a ocupar. Por tanto, con esta nueva fecha se acota aún más el espacio de tiempo en el que, como algún autor apunta, nuestro pintor pudo trasladarse a Roma en $1636^{7}$.

Velázquez es el pintor encargado de tasar los cuadros que quedaron entre los bienes del fallecido Juan de la Rea, Criado de Su Majestad ${ }^{8}$. Poco sabemos sobre éste personaje ${ }^{9}$, a no ser que coincida con el Juan de la Rea, Ujier de Saleta y Mayordomo del Estado de Cámara, que aparece documentado entre los fondos del Archivo del Palacio Real de Madrid ${ }^{10}$. De hacerlo es lógico pensar, siempre alrededor del contexto de palacio, en una cierta relación o trato próximo entre dos personajes, un ujier y un pintor, ambos siempre criados del monarca. Esta cercanía será posible a partir de octubre de 1623 , fecha en la que Velázquez recibe su nombramiento como pintor del rey ${ }^{11}$.

El 15 de diciembre de 1635 Pedro de Velasco, testamentario del ya difunto Juan de la Rea, pide licencia para hacer el inventario de sus bienes ${ }^{12}$. La petición es concedida y según la fórmula habitual, se cita a un teniente de corregidor, en este caso al licenciado Fernando de Salazar Velasco. Esa misma fecha Pedro de Velasco comenzó el inventario ${ }^{13}$. Tan sólo se anotaron «...seis sillas / de Vaqueta colorada...» $\mathrm{y}$ «... Tres taburetes de lo mismo viejos, bajos,...» ya que «...en este estado se quedo el dho ymbentario por este dia...».

5 Con fecha 16 de octubre de 1636, ratificado por la Junta el 24 del mismo. Véase Cruzada Villaamil, Gregorio: Anales de la vida y de las obras de Diego de Silva y Velázquez: escritos con ayuda de nuevos documentos. Madrid, 1885, p. 93.

6 Mes en el que se cumple el 18 aniversario del matrimonio entre los ya por entonces padres y abuelos Juana y Diego.

7 Sobre este asunto, véase Harris, Enriqueta: Velázquez en Roma. En «A.E.A.», t. XXXI, n. ${ }^{\circ} 123,1958$, pp. 185-192. Con especial atención a la nota n. ${ }^{\circ} 2$.

${ }^{8}$ A.H.P.M.; p. ${ }^{\circ} 4.916$, fols. $225-225$ v. Juan de la Rea o Larrea. Ante Joan de Xerez, escribano de S.M. y del N. ${ }^{\circ}$ de la Villa de Madrid. Este protocolo, cosido de antiguo, tiene en parte alterado su orden cronológico.

9 Se trataría seguramente del Juan de la Rea, que a propósito de la visita a Madrid del Cardenal D. Francisco Barberini en 1626, escribió Relación de todo lo sucedido en la Legacía del Ilustrísimo señor D. Francisco Barberino,... donde se refiere su jornada, desde Roma a esta Corte,... (s.I. y s.a.). Ver sobre esta visita J. Simón Díaz, La estancia del Cardenal Legado Francesco Barberini en Madrid el año 1626. En «A.I.E.M.», t. XVII, 1980, pp. 159-213.

Mas adelante aparecerá un Juan de la Rea, posiblemente hijo o familiar próximo, como religioso del convento de N. ${ }^{a}$ S. ${ }^{\text {a }}$ de la Merced, en Madrid.

${ }_{10}$ Archivo General de Palacio, Madrid; exp. pers.; Larrea, Juan de; ca 537/16: "Juró por Ujier de Saleta en 13 de octubre 1607. Promovido a Mayordomo del Estado de la Cámara en 1. ${ }^{\circ}$ de mayo de 1621 (Véase Mora, Jaime de)».

«Su Mag.' a hecho mrd. a Jü de la Rea del officio de / May" del Estado de la Cámara; y Juró en mis manos / en Veinte y dos de Abril Passado deste año. en esta / Conformidad se a de assentar desde el dho dia en los / Libros del bureo de Su Mag." En S" Gerónimo a 3 de Mayo de 1621 / El Conde de Olivares.

«El conde de Olivares. Sumiller de Corps. / q Ju de la Rea Juró Por may ${ }^{m o}$ del / Estado de la Cámara en xxy de Abril / de $M d=x x j . / »$.

Mora, Jaime de; $c^{a}$ 2.654/17: «Ju de la Rea, Joan de la Rea juró por Uger (sic) dę Saleta de Su M" en treçe de ott "I de 1607 as $^{\circ}$ en Bureo estando en él el Marqués de la Laguna /May" Mayor /». Al margen, "Fue Promovido a ma-/yordomo del Estado / de la $\mathrm{Cam}^{\text {ra }}$ en prim ${ }^{\text {ro }}$ / de mayo de 621 1». Por tanto, Juan de la Rea obtiene su primer cargo bajo el reinado de Felipe III y el segundo bajo Felipe IV, Recordemos que este último monarca subió al trono español el 31 de marzo de 1621, poco más de un mes antes de recibir Juan de la Rea su segundo cargo.

"Véase Zarco del Valle, M. R.: Documentos inéditos para la historia de las Bellas Artes en España. En «Colección de documentos inéditos para la historia de España por los señores Marqués de Miraflores y D. Miguel Salvá, individuos de la Academia de la Historia». Madrid, 1870, t. LV, p. 201.

12 A.H.P.M.; op. cit., fol. 73.

13 Ibidem, fol $73 \mathrm{v}$. 
Hasta dentro de tres meses no volvemos a tener noticias del proceso. Será el 14 de marzo de 1636 cuando se retome y continúe dicho inventario. Tras concluirlo, Pedro de Velasco como testamentario y Ana de Larrea como hija del fallecido ${ }^{14}$, firman el documento.

El 15 de abril de 1636 los tasadores realizan su trabajo ${ }^{15}$. Aparecen por orden documental Juana Pacheco ${ }^{16}$, Luis de Guadarrama ${ }^{17}$, Pedro de León ${ }^{18}$, Juan Mexía ${ }^{19}$ y Diego Velázquez ${ }^{20}$. Sólo vamos a transcribir aquí los bienes valorados por Juana y Diego, matrimonio que en definitiva abre y cierra toda la tasación ${ }^{21}$. Los bienes en general, atendiendo a su diferente naturaleza, son de poca consideración artística y económica.

Juana Pacheco, sin nombrar oficio alguno, aparece valorando «...los bienes muebles tocantes / a su tasación...». Este hecho es enormemente llamativo. Al final doña Juana, como aquí aparece, olvida escribir la primera vocal de su apellido en el momento de escribir su firma (Fig. 3) ${ }^{22}$.

Diego Velázquez «...Pintor del Rey Nro. $S^{r}$ aviendo Ju- / rado en forma de derecho tasso los bienes tocantes al / dho su oficio...». Los precios marcados por Velázquez varían entre 100 y 4 reales, lo que da una idea aproximada de la calidad de estas pinturas. Sobre ninguna aparece autoría o medidas ni se escribe si son originales, copias o si llevan algún tipo de marco. Su firma incluye sus apellidos de Silva Velázquez (Fig. 4).
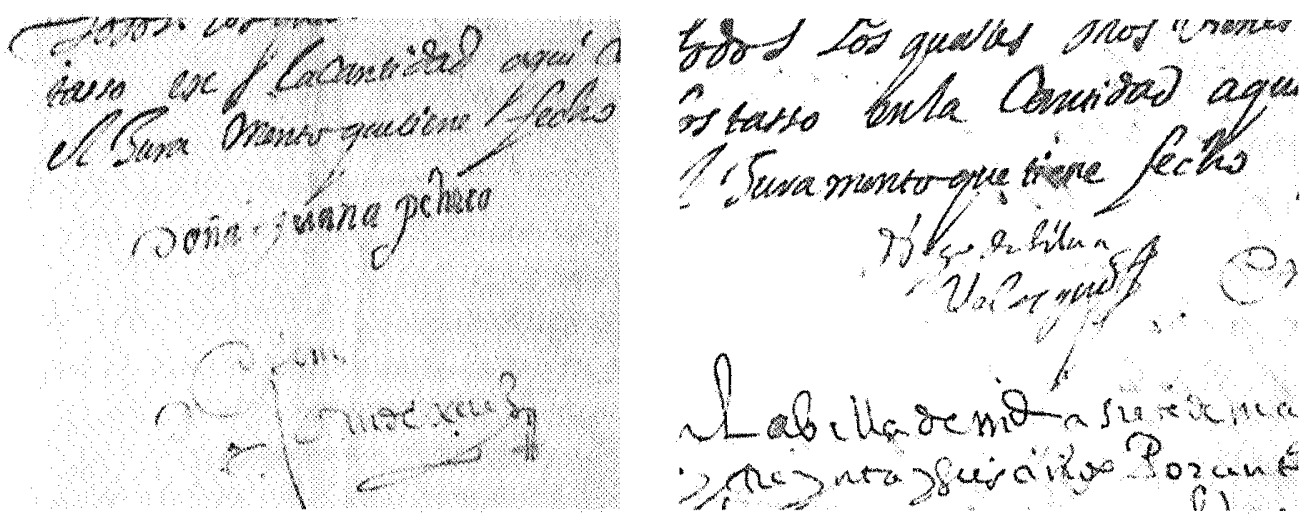

Figura 3. Firma de Juana Pacheco.

Figura 4. Firma de Diego Velázquez.

\footnotetext{
14 Será citada como tal, al final del documento.

15 Todos firman excepto Juan Mexía, del que se escribe «...y no firmó por no saber».

16 A.H.P.M.; op. cit., fols. 219-221.

17 Ibidem, fols. 222-222v. Maestro de tapicería.

18 Ibidem, fols. 223-223v. De oficio camero. .

19 Ibidem, fols. 224-224v. Ensamblador.

20 Ibidem fols. 225-225v.

21 Véase al final del artículo el apéndice documental.

22 Fotos del autor con permiso del A.H.P.M.
} 
$\mathrm{Al}$ mes siguiente se produce la retasa de algunos bienes. Efectivamente el 7 de mayo, Juan Mexía vuelve a comprobar el estado de dos bufetes de caoba. En esta ocasión baja su precio de veinte a doce ducados cada uno, debido a «...que están quebrados y maltratados» ${ }^{23}$.

Una vez terminada toda la tasación se produjo con fecha 17 de abril la puesta en venta, mediante pública almoneda, de todos los bienes señalados ${ }^{24}$. Lorenzo de Pinto, curador $a d$ lítem ${ }^{25}$ de los hijos menores de Juan de la Rea «...digo que Para hazer / la Partizion de sus bienes se a hecho inben- / tario y tasazion de sus bienes y es neze- / sario azar almoneda de ellos, suplico a $V \mathrm{md}$. I se dé lizenzia para que se haga la dha almoneda /... en cumplimiento del Auto del señor teniente por ante / el presente escrivano pressentes, el Padre fr. hernando I del Villar procurador del convento de nra. Señora de la / Mrd. por lo que toca al dho convento por el Padre fr. / Juan de la Rea Religiosso del dho convento $=y /$ por lo que le toca $=$ y Doña Ana de la Rea = y Pedro de /Velasco testamentario del dho difunto por Vos Bar- / tolome Martinez Pregonero publico se hiço almone- / da de los bienes del dho Juan de la Rea ya difunto / en las cassas donde vivio y murio que son las del / Licenciado Don Francisco de los herreros en la forma / siguiente...».

No era costumbre en la época que un tasador apareciera en la almoneda de unos determinados bienes adquiriendo algún objeto valorado con anterioridad por él mismo. Aunque éste no sea ese caso estricto, sí hay que comentar que Velázquez aparece entre los compradores en esta almoneda ${ }^{26}$. Las personas que aparecen comprando algún objeto son Pedro Velasco «el mozo» ${ }^{27}$, d. ${ }^{a}$ María de Losada, d. Gutierre (sic.) de Terán, Francisco García, Ana Ruiz, sr. licenciado Serrano, María Tomé, d. Antonio de la Riva, el contador Gamarro, d. Francisco de los Herreros ${ }^{28}$, Prudencio Fernández, Juan de la Llana Terán, Juan de Oribe, Julián de los Herreros, el licenciado Valle, Manuel de los Reyes, Manuel de San (sic.), Diego Velázquez, pintor, Jusepe Corilla, platero, d. ${ }^{a}$ María de Baeza, el convento de la Merced, d. ${ }^{a}$ Margarita de Saldaña y el doctor Encinas. Firma la almoneda, estando siempre presente, Ana Antonia de la Rea.

La adquisición de Velázquez se centra sobre los siguientes objetos:

- «En Diego Belazquez Pintor se / rremato Una tapicería de Bos- / caxe que tiene seis Paños / y medio a y çiento y doçe anas / a siete Reales el ana que montan / seteçientos y ochenta y quatro Rs. 784 / (Otra pág.)

- En el dicho tres paños de ta- / piceria de montería de quatro / anas de cayda que tienen cin- / cuenta y ocho anas a ocho / rreales el ana montan qua- / troçientos y sesenta y quatro Rs. $464^{30}$. I

- Una tabla de manteles Reales / en cinco ducados se le remato $055^{31}$,

23 A.H.P.M.; op. cit., fol, $225 \mathrm{v}$. Esta retasa curiosamente es posterior a la fecha de la almoneda.

24 Ibidem, fols. 228-233. La almoneda dice hacerse el 17 ó 16 de abril, dependiendo de qué parte del documento tratemos.

${ }^{25}$ Es la persona nombrada por un juez para continuar los pleitos y defender los derechos de un menor, representándole a su vez.

${ }_{36}$ Sobre otras apariciones suyas como comprador en almoneda, véase J. L. Barrio Moya, Un documento sobre adquisiciones de Velázquez. En «Archivo Español de Arte», t. XLIX, n. ${ }^{195}$, julio-septiembre 1976, varia, pp. $327-328$

27 Quizás hijo de Pedro de Velasco, testamentario del difunto.

28 Licenciado. Dueño de las casas donde vivió Juan de la Rea.

29 Luis de Guadarrama, al tasar dicha tapicería, no especifica la cantidad total.

30 Idem.

31 Se tasaron en 60 rs. 
- En el dicho Una sabana nueba / de Ruan En $V^{t e}$ y ocho Reales 028 I

- En el dicho Una tabla de man- / teles Limaniscos En V $V^{\text {te }}$ Rs $020 /$

- En el dicho Se rremato Una tabla / de manteles limaniscos biexos / y Rota En Seys rreales $006^{32}$ /

- En el dicho Un perol de açofar / En diez y seis Reales $016^{33} /$

- En el dicho Una sarten En dos Rs. $002{ }^{34} 1$

- En el dicho Un bufete de nogal / En treynta Reales 030 /

- En el dicho, una salba de Plata / dorada que Pesa tres marcos / una onça y quatro ochabas a / sesenta y cinco rreales montan / doçientos (sic) y siete Reales / (Otra pág.)

- de la echura de la salba y oro / veynte y dos $R^{S} 022 »$.

Por otro lado, una vez revisados los bienes que se inventariaron de manera conjunta tras la muerte del matrimonio Velázquez en agosto de $1660^{35}$, es difícil establecer una identificación exacta entre alguno de dichos bienes con los adquiridos en la almoneda de Juan de la Rea. Por la poca relevancia en sí de estos objetos y por la ligera descripción que tras la muerte del pintor se hace de ellos, resulta complicado indicar la naturaleza de los que aquí intere$\operatorname{san}^{36}$.

Una vez vendida prácticamente la totalidad de los bienes, continúa la almoneda durante el 7 de mayo con la venta de algunos enseres más.

Al año siguiente, el 11 de mayo de 1637, «... d. ${ }^{a}$ Ana de la / rrea Por $q^{\text {ta }}$ de su ligitima (sic) Tomo los bie- / nes que adelante se ara mençion...», y es aquí la primera y única vez que se establece relación directa entre el fallecido y d. ${ }^{a}$ Ana, aludiendo al «... imbentario que se hizo por muerte del / dho su padre...».

La última referencia documental es del 9 de julio de 1637. Ana cobra un dinero que se debía a su padre, haciendo referencia en estos términos: «... puso más por ym-/ bentario seteçientos y ocho Re- / ales que tiene en su Poder que / cobro Alonso Mellado urgel (sic) de / Camara de Su mag ${ }^{37}$ y testementa- / rio del dho Juan de la rrea de / dos terçios de los gaxes que se le de-/ bian a el dicho Juan de la rrea quel (sic)/ uno es de fin del año Pasado de / seysçientos y treynta y quatro /...».

Esta breve aportación documental permite contextualizar bajo unos parámetros concretos de espacio y tiempo una labor poco habitual en tan genial artista.

FERNANDO LÓPEZ SÁNCHEZ Universidad Complutense de Madrid

32 Tasados en 8 rs.

33 Tasado y vendido en la misma cantidad.

34 Tasada en 3 rs.

35 Véase Sánchez Cantón, F. J.: Cómo vivía Velázquez. Inventario descubierto por D. F. Rodríguez Marín. En «A.E.A.», 15, 1942, pp. 69-91. (A.H.P.M., P. ${ }^{\circ} 8.137$, fols. 693-709).

${ }^{36}$ Sólo podríamos relacionar entre ambos inventarios, como posibles objetos identificados: dos tablas de manteles limaniscos (sic), con «tres tablas de manteles alemaniscos traydos» (fol. 701 de los bienes tras la muerte de Velázquez); un perol de azófar, con «tres Peroles Pequeños» (fol. 708); una sartén, con «çinco sartenes» (fol. 708); y un bufete de nogal, con «Un bufete de nogal sin cajón» (fol. 694). De las tapicerías, no hay posible relación.

37 Se refiere a Alonso Mellado, Ujier de Cámara de S.M. y criado del Rey. Antes, mozo y ayuda de la tapicería. Este personaje de vida longeva trabajó bajo los reinados de Felipe II, Felipe III y Felipe IV. Su único perfil biográfico lo esboza en su interesante trabajo Cruz Yábar, M. ${ }^{a}$ Teresa: La Tapicería en madrid (1570-1640). Madrid, 1996, pp. 178-179. Sobre su expediente personal, pp. 454-455. 